\title{
Elementary abelian subgroups in some special $p$-groups
}

\author{
Xingzhong $\mathrm{Xu}^{1,2}$
}

\begin{abstract}
Let $P$ be a finite $p$-group and $p$ be an odd prime. Let $\mathcal{A}_{p}(P)_{>2}$ be a poset consisting of elementary abelian subgroups of rank at least 2 . If the derived subgroup $P^{\prime} \cong C_{p} \times C_{p}$, then the spheres occurring in $\mathcal{A}_{p}(P)_{\geq 2}$ all have the same dimension.
\end{abstract}

Key Words: posets; subgroup complexes.

\section{Introduction}

Let $P$ be a finite $p$-group, and the poset of all nontrivial elementary abelian $p$ subgroup of $P$ is denoted by $\mathcal{A}_{p}(P)$. In [4, Bouc and Thévenaz focused on subposet $\mathcal{A}_{p}(P)_{\geq 2}$ consisting of elementary abelian subgroups of rank at least 2 . And they determined the homotopy type of the complex $\mathcal{A}_{p}(P)_{\geq 2}$ : a wedge of spheres (of possibly different dimensions). We refer the reader to [9] for more details about $p$-subgroups complex. After computer calculations, Bouc and Thévenaz posed the following questions in 44.

Question 1.1. (Bouc, Thévenaz) Let $P$ be a p-group. Do the spheres occurring in $\mathcal{A}_{p}(P)_{\geq 2}$ all have the same dimension if $p$ is odd? Does one get only two consecutive dimensions if $p=2$ ?

In [3], Bornand proved that Question 1.1 has a positive answer if $P$ has a cyclic derived subgroup. Recently, [6] provided a negative answer to Question 1.1. But determining the dimensions of those spheres of $\mathcal{A}_{p}(P)_{\geq 2}$ is still an interesting question, thus the following theorem is the main result in this short paper.

Theorem 1.2. Let $P$ be a finite $p$-group and $p$ be an odd prime. If the derived subgroup $P^{\prime} \cong C_{p} \times C_{p}$, then the spheres occurring in $\mathcal{A}_{p}(P)_{\geq 2}$ all have the same dimension.

Structure of the paper : After recalling the basic properties of finite $p$-group with cyclic derived group in Section 2, we give a proof of Theorem 1.2 in Section 3.

\section{Notation and finite $p$-groups}

In this section, let $p$ be an odd prime. We refer the reader to [7, 8] for the theory of finite group and topology respectively. Now, recall that a minimal nonabelian

\footnotetext{
* Date: 05/11/2017.

1. Department of Mathematics, Hubei University, Wuhan, 430062, China

2. Departament de Matemàtiques, Universitat Autònoma de Barcelona, E-08193 Bellaterra,

Xingzhong Xu's E-mail: xuxingzhong407@mat.uab.cat; xuxingzhong407@126.com

Supported by NSFC grant (11501183).
} Spain 
$p$-group means that all its proper subgroups are abelian. Let $P$ be a minimal nonabelian $p$-group. Then $P$ is one of the following groups:

$$
\begin{aligned}
& S(n, m, 0):=\left\langle x, y \mid x^{p^{n}}=y^{p^{m}}=1,[x, y]=x^{p^{n-1}}\right\rangle(n \geq 2) ; \\
& S(n, m, 1):=\left\langle x, y, z \mid x^{p^{n}}=y^{p^{m}}=z^{p}=1,[x, y]=z,[z, x]=[z, y]=1\right\rangle .
\end{aligned}
$$

Here, $S(n, 1,1) \cong S(1, n, 1)$. One can find these results about nonabelian $p$-groups in [1, 10. In particular, $S(2,1,0)$ and $S(1,1,1)$ are extraspecial $p$-groups. And they always are written as $p_{-}^{1+2}, p_{+}^{1+2}$ respectively.

Recall that a group $H$ is said to be the central product of its normal subgroups $H_{1}, H_{2} \ldots, H_{n}$ if $H=H_{1} H_{2} \cdots H_{n},\left[H_{i}, H_{j}\right]=1$ for $i \neq j$, and $H_{i} \cap \prod_{j \neq i} H_{j} \leq$ $Z(H)$ for all $i$.

The classification of finite $p$-groups with derived subgroup of prime order, traces back to S. Blackburn.

Proposition 2.1. 2, Proposition 9] Let $p$ be an odd prime and $S$ be a finite $p$ group. If $\left|S^{\prime}\right|=p$, then $S$ is a central product as following types:

$$
S \cong S_{1} * S_{2} * \cdots * S_{l} * A
$$

where $S_{i} \cong S\left(n_{i}, m_{i}, 0\right)$ or $S\left(n_{i}, m_{i}, 1\right)$ and $A$ is a finite abelian $p$-group. Here, $S_{i} \cap\left(\prod_{j \neq i} S_{j}\right) A=S^{\prime}$ and $A \cap \prod_{i} S_{i} \leq S^{\prime}$.

Moreover, if $Z(S)$ is cyclic, we get the following corollary which will be used to prove Theorem 1.2 in Section 3.

Corollary 2.2. Let $p$ be an odd prime and $S$ be a finite $p$-group. If $\left|S^{\prime}\right|=p$ and $Z(S)$ is cyclic, then $S$ is a central product as following types:

$$
S \cong S(n, 1,0) * p_{+}^{1+2} * \cdots * p_{+}^{1+2} \text {, or } S \cong p_{+}^{1+2} * p_{+}^{1+2} * \cdots * p_{+}^{1+2} .
$$

\section{The proof of the main theorem}

In this section, we give a proof of Theorem 1.2.

Theorem 3.1. Let $P$ be a finite $p$-group and $p$ be an odd prime. If $P^{\prime} \cong C_{p} \times C_{p}$, then the spheres occurring in $\mathcal{A}_{p}(P)_{\geq 2}$ all have the same dimension.

Proof. Suppose that the spheres occurring in $\mathcal{A}_{p}(P)_{\geq 2}$ do not have the same dimension, then we want to get a contradiction to prove the theorem.

Since $P^{\prime} \cong C_{p} \times C_{p}$, we can set $P^{\prime}=\langle a\rangle \times\langle b\rangle$ and $|a|=|b|=p$. If $Z(P)$ is not cyclic, then $\Delta\left(\mathcal{A}_{p}(P)_{\geq 2}\right)$ is contractible. That is a contradiction. Hence, $Z(P)$ is cyclic and we write $Z:=\langle a\rangle$ for the unique subgroup of order $p$ in $Z(P)$ because $Z(P) \cap P^{\prime} \geq Z$. Moreover, we can assume $b \notin Z(P)$.

Step 1. We set $E_{0}=P^{\prime}$ and

$$
M:=C_{P}\left(E_{0}\right):=\left\langle a, b, y_{1}, y_{2}, \ldots, y_{n}\right\rangle
$$

for some $y_{1}, y_{2}, \ldots, y_{n} \in P$. Let $\chi:=\left\{E_{1}, E_{2}, \ldots, E_{k}\right\}$ be the subset of $\mathcal{A}_{p}(P)_{>Z}$ of all elementary abelian subgroups of rank 2 not contained in $M$. Suppose $\chi$ is empty set, thus $\Delta\left(\mathcal{A}_{p}(P)_{\geq 2}\right)$ is contractible. That is a contradiction. Hence $\chi$ is not empty set. Then there exists $x \in E_{1}-M$, it implies that

$$
[x, b] \neq 1 \text {, and }|x|=p \text {. }
$$


We assert that $[x, b]=a^{i}$ for some $1 \leq i \leq p-1$. If $1 \neq[x, b]=b a^{i}$, then we can see $\left[x, b a^{i}\right]=b a^{i}$ because $a \in Z(P)$. That is a contradiction. Hence, $[x, b]=a^{i}$. Therefore, we can assume that

$$
[x, b]=a .
$$

Step 2. We assert that $M^{\prime} \leq\langle a\rangle$. Since $M^{\prime} \leq P^{\prime}=\langle a, b\rangle$, for each $y_{i}, y_{j}$, we have

$$
\left[y_{i}, y_{j}\right]=a^{\varepsilon} b^{\epsilon} .
$$

where $\varepsilon, \epsilon \in \mathbb{N}$. We can see that

$$
\left[x,\left[y_{i}, y_{j}\right]\right]=\left[x, a^{\varepsilon} b^{\epsilon}\right]=a^{\epsilon} .
$$

But $\left[\left[x, y_{i}\right], y_{j}\right]=1$ and $\left[\left[y_{j}, x\right], y_{i}\right]=1$ because $\left[x, y_{i}\right],\left[y_{j}, x\right] \in P^{\prime}=\langle a, b\rangle=E_{0}$ and $y_{i}, y_{j} \in M=C_{P}\left(E_{0}\right)$. By the Hall-Witt identity, we have $\left[x,\left[y_{i}, y_{j}\right]\right]=1$. It implies $b^{\epsilon}=1$. So $\epsilon \equiv 0(\bmod p)$. Hence

$$
\left[y_{i}, y_{j}\right] \in\langle a\rangle
$$

for every $y_{i}, y_{j}$. Because $\langle a, b\rangle \leq Z(M)$, thus $M^{\prime} \leq\langle a\rangle$.

By the definition of $E_{i}$, we can set $E_{i}=\left\langle x t_{i}, a\right\rangle$ where $t_{i} \in M$ such that

$$
M E_{i}=P .
$$

In other words, set $F_{i}=\left\langle x t_{i}\right\rangle$ such that

$$
P=M \rtimes F_{i} .
$$

We consider the groups $C_{M}\left(E_{i}\right)$. We set $P_{i}=C_{M}\left(E_{i}\right)$. Since $P_{i} \leq M$, thus $P_{i}^{\prime} \leq M^{\prime} \leq\langle a\rangle$. Therefore, we have $P_{i}^{\prime} \cong C_{p}$ or $P^{\prime}=1$.

Step 3. By the proof of [4, Thoerem 14.1], we can see that

$$
\Delta\left(\mathcal{A}_{p}(P)_{\geq 2}\right) \simeq \bigvee_{i=1}^{k} \Delta\left(\mathcal{A}_{p}\left(P_{i}\right)_{\geq 2}\right)
$$

Since the spheres occurring in $\mathcal{A}_{p}(P)_{\geq 2}$ do not have the same dimension, thus we can suppose that the dimension of the sphere occurring in $\mathcal{A}_{p}\left(P_{1}\right)_{\geq 2}$ do not equal the dimension of the sphere occurring in $\mathcal{A}_{p}\left(P_{2}\right)_{\geq 2}$, and $\Delta\left(\mathcal{A}_{p}\left(P_{1}\right)_{\geq 2}\right)$ and $\Delta\left(\mathcal{A}_{p}\left(P_{2}\right)_{\geq 2}\right)$ are not contractible.

By Step 2 , we can see that $P_{1}^{\prime} \cong P_{2}^{\prime} \cong C_{p}$ and $Z\left(P_{1}\right), Z\left(P_{2}\right)$ are cyclic. Here, we can set $P_{1}=C_{M}\left(E_{1}\right)=C_{M}(\langle x, a\rangle)$ and $P_{2}=C_{M}\left(E_{2}\right)=C_{M}(\langle x t, a\rangle)$ where $1=t_{1}, t=t_{2} \in M$. By Corollary 2.2 , we can set

$$
P_{1} \cong S_{1} * S_{2} * \cdots * S_{l} * A
$$

where $S_{i} \cong S\left(n_{i}, m_{i}, 0\right)$ or $S\left(n_{i}, m_{i}, 1\right)$. Since $Z\left(P_{1}\right)$ is cyclic, we can set

$$
P_{1} \cong S_{1} * S_{2} * \cdots * S_{l} \cong \underbrace{S\left(n_{1}, 1,0\right) * p_{+}^{1+2} * p_{+}^{1+2} * \cdots * p_{+}^{1+2}}_{l}
$$

or

$$
P_{1} \cong S_{1} * S_{2} * \cdots * S_{l} \cong \underbrace{p_{+}^{1+2} * p_{+}^{1+2} * \cdots * p_{+}^{1+2}}_{l} .
$$

If $P_{1} \cong S\left(n_{1}, 1,0\right) * p_{+}^{1+2} * p_{+}^{1+2} * \cdots * p_{+}^{1+2}$, we can set $P_{1}=\left\langle a, u_{1}, u_{2}, \ldots, u_{2 l}\right\rangle$ such that

$$
\left\langle a, u_{2 i-1}, u_{2 i}\right\rangle \cong S_{i} \text {, and } u_{1}^{p^{n_{1}-1}}=a .
$$


We can see that every elementary abelian subgroups of rank at least 2 must contain $\left\langle u_{1}^{p^{n_{1}-1}}, u_{2}\right\rangle$. Hence, $\Delta\left(\mathcal{A}_{p}\left(P_{1}\right)_{\geq 2}\right)$ is contractible. Hence,

$$
P_{1} \cong S_{1} * S_{2} * \cdots * S_{l} \cong \underbrace{p_{+}^{1+2} * p_{+}^{1+2} * \cdots * p_{+}^{1+2}}_{l} .
$$

Similarly, we can set

$$
P_{2} \cong R_{1} * R_{2} * \cdots * R_{s} \cong \underbrace{p_{+}^{1+2} * p_{+}^{1+2} * \cdots * p_{+}^{1+2}}_{s} .
$$

Here, $n_{1}, n_{2}, l, s \in \mathbb{N}$. And we can see $P_{1}=\left\langle a, u_{1}, u_{2}, \ldots, u_{2 l}\right\rangle$ such that

$$
\left\langle a, u_{2 i-1}, u_{2 i}\right\rangle \cong S_{i}
$$

And we also can set $P_{2}=\left\langle a, v_{1}, v_{2}, \ldots, v_{2 s}\right\rangle$ such that

$$
\left\langle a, v_{2 i-1}, v_{2 i}\right\rangle \cong R_{i} \text {. }
$$

By [3, Proposition 4.7], we can see that the dimension of the sphere occurring in $\mathcal{A}_{p}\left(P_{1}\right)_{\geq 2}$ is $l-1$ and the dimension of the sphere occurring in $\mathcal{A}_{p}\left(P_{2}\right)_{\geq 2}$ is $s-1$. Since $s \neq l$, we can assume that $s<l$.

Since $P_{1}, P_{2} \leq M$ and $\left|M^{\prime}\right|=p$, we can assume that $\left\langle u_{2 i-1}, u_{2 i}\right\rangle \cap P_{2} \leq$ $Z\left(\left\langle P_{1}, P_{2}\right\rangle\right)$ for some $i$ because $s<l$. Hence $\left[x t, u_{2 i-1}\right] \neq 1$ and $\left[x t, u_{2 i}\right] \neq 1$. But $\left[x, u_{2 i-1}\right]=1$ and $\left[x, u_{2 i}\right]=1$ (because $u_{2 i-1}, u_{2 i} \in P_{1}=C_{M}\left(E_{1}\right)=C_{M}(\langle x, a\rangle)$ ), thus $\left[t, u_{2 i-1}\right] \neq 1$ and $\left[t, u_{2 i}\right] \neq 1$.

Moreover, we can set

$$
\begin{gathered}
{\left[t, u_{2 i-1}\right]=a^{\varepsilon_{1}} \neq 1,} \\
{\left[t, u_{2 i}\right]=a^{\epsilon_{1}} \neq 1}
\end{gathered}
$$

for some numbers $\varepsilon_{1}, \epsilon_{1} \in \mathbb{N}$. There exist two numbers $\left(\varepsilon_{1}^{\prime}, \epsilon_{1}^{\prime}\right) \neq(0,0)(\bmod p)$ such that $\varepsilon_{1} \varepsilon_{1}^{\prime}+\epsilon_{1} \epsilon_{1}^{\prime} \equiv 0(\bmod p)$. Hence

$$
\left[t, u_{2 i-1}^{\varepsilon_{1}^{\prime}} u_{2 i}^{\epsilon_{1}^{\prime}}\right]=a^{\varepsilon_{1} \varepsilon_{1}^{\prime}+\epsilon_{1} \epsilon_{1}^{\prime}}=1 .
$$

Here, $u_{2 i-1}^{\varepsilon_{1}^{\prime}} u_{2 i}^{\epsilon_{1}^{\prime}} \neq 1$. It implies $1 \neq u_{2 i-1}^{\varepsilon_{1}^{\prime}} u_{2 i}^{\epsilon_{1}^{\prime}} \in P_{2}$. But $\left\langle u_{2 i-1}, u_{2 i}\right\rangle \cap P_{2} \leq$ $Z\left(\left\langle P_{1}, P_{2}\right\rangle\right)$, thus $u_{2 i-1}^{\varepsilon_{1}^{\prime}} u_{2 i}^{\epsilon_{1}^{\prime}}=1$. That is a contradiction.

\section{ACKNOWLEDGMENTS}

The author would like to thank Prof. C. Broto for his constant encouragement in Barcelona in Spain.

\section{REFERENCES}

[1] N. Blackburn, Generalizations of certain elementary theorems on p-groups, Proc. London Math. Soc. 11(3), 122 (1961), 1-22.

[2] S. Blackburn, Groups of prime power order with derived subgroup of prime order, J.Alg, 219 (1999), 625-657.

[3] D. Bornand, Elementary abelian subgroups in p-groups with a cyclic derived subgroup, J. Alg. 335 (7) (2011), 301-318.

[4] S. Bouc, J. Thévenaz, The poset of elementary abelian subgroups of rank at least 2, Monogr. Enseign. Math. 40, (2008) 41-45.

[5] W. Dwyer, H. Henn, Homotopy theoretic methods in group cohomology, Advanced courses in mathematics. CRM Barcelona, Birkhäuser Verlag (2001)

[6] F. Fumagalli, J. Shareshian, Truncated Quillen complexes of p-group, J. Algebr. Comb. 40 (2014), 771-784.

[7] W. Gorenstein, Finite groups, Chelsea, London (1968)

[8] A. Hatcher, Algebraic topology, Cambridge Univ. Press (2002) 
[9] D. Quillen: Homotopy properties of the poset of nontrivial p-subgroups of a group. Adv. Math. 28(2) (1978), 101-128.

[10] L. Rédei, Das schiefe Product in der Gruppentheorie, Comment. Math. Helvet. 20 (1947), 225-267. 